\title{
Properties and Behaviour of a Virus Depending for its Multiplication on Another
}

\author{
By B. KASSANIS \\ Rothamsted Experimental Station, IIarpenden, Ilertfordshire
}

(Received 19 July 1961)

\begin{abstract}
SUMMARY
The Rothamsted culture of tobacco necrosis virus contains two scrologically unrelated viruses one of which, called the 'satellite virus' (SV), causes no lesions and multiplies detectably only when the other (TNV) is present. It decreases the size of necrotic local lesions formed by TNV. Inocula containing both viruses gave the same, fewer or more lesions than inocula containing only TNV ; the results depended on the ratio of the two viruses in the mixed inoculum, on the species and age of the test plants and, particularly, on the temperature at which the plants were kept after the inoculation. The concentration of TNV decreased when SV was also present; the extent of the decrease depended on the ratio of the two viruses in the inoculum and other factors. Inoculation with SV 5 days before inoculation with 'TNV still decreased lesion size and led to the multiplication of SV. Phenol-disrupted SV affected lesion size only when inoculation with TNV followed within a few hours, but when inoculation with TNV was delayed by up to 2 days, SV sometimes multiplied detectably. SV has the smallest particle reported for any plant virus; diameter c. $17 \mathrm{~m} \mu$ and weight equivalent to a molecular weight of about $1.9 \times 10^{8}$, of which $20 \%$ is nucleic acid. It is exceptionally stable, retaining infectivity after 17 years at $3^{\circ}$ and after heating for $10 \mathrm{~min}$. at $90^{\circ}$. TNV was much more susceptible to inactivation by licat and by ultraviolct light than SV. The two differed greatly in electrophoretic: mobility and were readily scparated from mixed preparations by electrophoresis.
\end{abstract}

\section{INTRODLCTION}

The Rothamsted culture of tobacco nccrosis virus was long known to contain spherical particles of two sizes (Bawden \& Pirie, 1942, 1950) but the significance of this was not understood until they were found to be serologically unrelated viruses, the smaller of which multiplies detectably only in the presence of the larger. The simultancous multiplication of the two produces smaller necrotic local lesions in French bean than when the large particles are multiplying alone; and the ratio of large to small lesions falls as the proportion of small particles in the inoculum increascs; a grcat excess of small particles can decrease the number of lesions (Kassanis \& Nixon, 1960, 1961). The experiments now to be described show other effects of interaction between the two viruses and how their interactions depend on the species of host plant and changes in the environment. Also, by using the 
proportion of large to small lesions formed by a mixed inoculum as a method of assaying small particle infectivity some of the properties of the small virus particles have been determined. For need of a better name, the virus with small particles was called 'satcllite virus' ( $\mathrm{SV}$ ) because it is always in association with and dependent on the large virus particles.

\section{METHODS}

The virus inocula were prepared as described by Kassanis \& Nixon (1961). The satellite virus (SV) was obtained from the top zone after centrifugation in sucrose gradicnts of purified preparations of an isolate of the Rothamsted culture. The ratio of small to large particles varied in different preparations, depending on the season and the age of the tobacco plants, from 10:1 to $250: 1$. The total yield of virus was smaller in summer than winter. The particles of two sizes separated into zones best when the preparations contained no more than $2 \mathrm{mg} . / \mathrm{ml}$. of virus. Plants inoculated with purified preparations of SV produced no lesions and the virus did not multiply detectably in them. SV can be activated by two tobaceo necrosis viruses, TNVa or 'TNVb (Kassanis \& Nixon, 1961), but in the present work $\mathrm{TNVb}$ was mainly used. All virus dilutions were in water. Purification, infectivity tests and particle counts with the electron microscope were made as before (Kassanis \& Nixon, 1961).

The proportion of SV surviving after various treatments was measured by comparing its effect on the lesion formed by 'INVb with untreated $\mathrm{SV}$ of known concentration. The treated $S V$ was inoculated at one dilution and the untreated at 4 dilutions, after mixing with constant amounts of TNVb (enough to give about 20) 60) lesions ihalf bean leaf). The numbers of large lesions were plotted against the concentration of untreated $\mathrm{SV}$, and the concentration of $\mathrm{SV}$ remaining active in the treated preparation was estimated by interpolation. 'This method assumes that loss of the ability of SV to affect the size of lesions produced by TNVb coincides with loss of infectivity; there is evidence to show that the assumption is correct.

An antiserum to $\mathrm{SV}$ was prepared by injecting a rabbit with $\mathrm{SV}$ purified by two successive centrifugations in sucrose gradients. A total of $0 \cdot 7 \mathrm{mg}$. of $\mathrm{SV}$ was injected into a rabbit in three injections; the first was intravenous and a month later two intramuscular injections were given on successive days. The virus injected intramuseularly was cmulsified in Bacto Adjuvant, Complete (Difoo Laboratories). The rabbit was bled a month after the last injection and when titrated against SV at $20 \mathrm{mg} . / \mathrm{l}$. the serum precipitated when diluted 1/400. This antiserum did not precipitate TNVa or TNVb; nor did antisera to these viruses precipitate $S V$, thus confirming earlier evidence that the large and small virus particles are serologically unrelited (Kassanis \& Nixon, 1961).

The concentration of purified virus preparations was estimated from measuring their optical density at $260 \mathrm{~m} \mu$, and the concentration of the virus in sap by determining the highest dilution at which a specific precipitate was produced with the homologous antiserum. The sap used in serological tests was frozen, thawed, left for a day at $20^{\circ}$, centrifuged and heated at $45^{\circ}$ for $10 \mathrm{~min}$. before again being contrifuged at $8000 \mathrm{~g}$. When the increase of virus in bean lcaves was investigated, the water lost from the necrotic leaves was estimated by comparing their water content with that of uninoculated lcaves and the loss was compensated for by 
adding an appropriate amount to the leaves when they were ground before extracting the sap. Most of the experiments were made between autumn and spring when the glasshouse temperature fluctuated between $16^{\circ}$ and $20^{\circ}$. The temperature in summer fluctuated between 20 and $30^{\circ}$, which sometimes was too high for virus multiplication.

\section{RFSITLTS}

Interference during lesion formation

The necrotic local lesions formed by inocula containing only TNVa or TNVb were all large, but inocula that also contained SV gave some small lesions. the number of which depended on the amount of SV in the inoculum (Fig. 1). The total number of lesions (large and small) formed by mixed inocula (constant TNVa or ' $\mathrm{TNV}$ b and varying amounts of $\mathrm{SV}$ ) was sometimes the same over a wide range of concentrations of SV (Table 3 in Kassanis \& Nixon, 1961). sometimes more and sometimes fewer (Fig, 1), depending on the age and species of the inorulated plants and their growing conditions before and after inoculation. A decrease was usual only with high concentrations of $S V$ and when all the lesions were small, but in

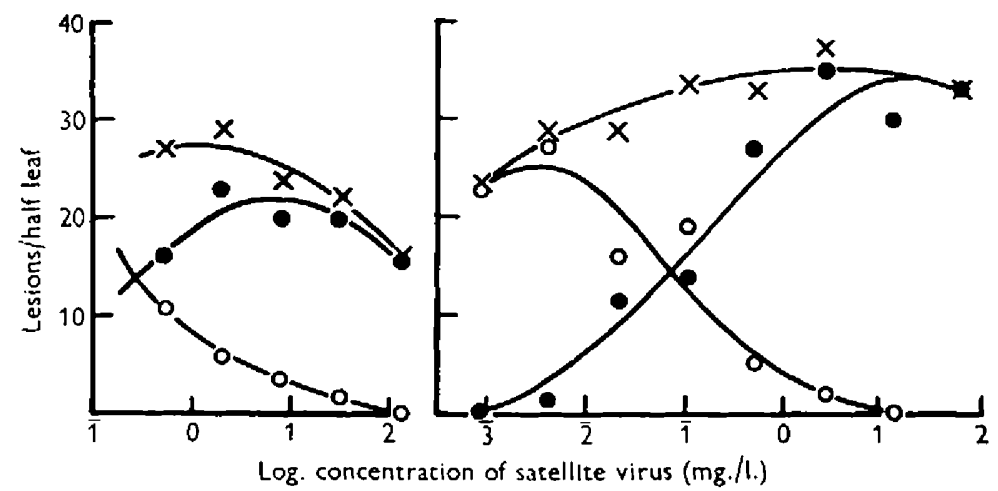

(a)

(b)

lig. 1. Ifesions/half leaf of French bean inoculated with mixtures of constant TNVb $(0 \cdot 3$ mgr. $/ 1$.$) and different anounts of SV. x-x$, total number of lesions; $O-O$, large lesions; - small lesions. $(a)$ and $(b)$, experiments made at differcnt times of the year.

summer it happened with lower concentrations of SV than in winter. Increases occurred mostly in winter and only when the ratios of the two viruses were such that not all lesions were small.

'The number of lesions produced by a given inoculum of tobacco necrosis virus depends on the temperature at which the plants are kept (Kassanis, 1952). An experiment was therefore made to find whether the tempernture also affected the extent to which TNVb and SV interacted. Bean plants were inoculated with constant amounts of ' $\mathrm{TNVb}$ mixed with varying amounts of $\mathrm{SV}$ and placed in glass clambers kept at $17^{\circ}, 22^{\circ}$ and $26^{\circ}$. The lesions appeared 1 day sooncr at $26^{\circ}$ than at the lower temperatures, but were fewer and all large. The highest concentration of SV decreased lesion numbers at all temperatures, and did so more at higher than at lower temperatures (Table 1 ). It is interesting that, with $1 \mathrm{mg} . / \mathrm{l}$. of SV, the total number of lesions decreased at $26^{\circ}$, but increased at $22^{\circ}$ and even more at $17^{\circ}$. 
These results strongly suggest that the interactions between $\mathrm{TNVb}$ and $\mathrm{SV}$ differ at different seasons mainly because of difference in temperature.

From these results it can be argued that, for a lesion to form, at least one of the susceptible sites (situated in one or more neighbouring cells) has to be occupied by TNVb. But when the concentration of SV is high or when the susceptible sites are few (by keeping the plants at a high temperature after inoculation or by other treatments), then the chances are that all the sites will be occupied by $\mathrm{SV}$ and the Iesion will not be formed. The fact that with susceptible plants kept at rclatively low temperatures considerably higlier concentrations of SV were needed to decrease the total number of lesions suggests that each infectable centre may contain several susceptible sites.

Table 1. Effect of temperature on number of large and small lesions formed by tobacco necrosis virus $(T N V b)$ simultaneously inoculated with satellit evirus $(S V)$ at different concentrations

\begin{tabular}{|c|c|c|c|c|c|c|c|c|c|}
\hline \multirow{3}{*}{$\begin{array}{l}\text { Con- } \\
\text { centra- } \\
\text { tion } \\
\text { of SV* } \\
\text { (mg./l.) }\end{array}$} & \multicolumn{3}{|c|}{ Small lesions } & \multicolumn{3}{|c|}{ Large lesions } & \multicolumn{3}{|c|}{$\begin{array}{c}\text { Total of } \\
\text { small + large lesions }\end{array}$} \\
\hline & $17^{\circ}$ & $22^{\circ}$ & $20^{\circ}$ & $17^{\circ}$ & $22^{\circ}$ & $26^{\circ}$ & $17^{\circ}$ & $22^{\circ}$ & $26^{\circ}$ \\
\hline & \multicolumn{9}{|c|}{ Number of lesions from 8 bean-half leaves } \\
\hline 25 & 227 & 93 & 0 & 0 & 0 & 4 & 227 & 93 & 4 \\
\hline 5 & 015 & 614 & 0 & 94 & 4 & 22 & 1009 & 018 & 22 \\
\hline I & 1097 & 522 & o & 118 & 83 & 36 & 1210 & 5.55 & 86 \\
\hline 0.2 & 333 & 299 & 0 & 259 & 189 & 74 & 502 & 488 & 74 \\
\hline 0.04 & 139 & 116 & 0 & 558 & 884 & 180 & 697 & 500 & 180 \\
\hline 0.008 & 38 & 13 & 0 & 513 & 341 & 137 & 551 & 354 & 137 \\
\hline
\end{tabular}

The interaction between $\mathrm{TNVb}$ and $\mathrm{SV}$ is also influenced by the age of the plants and the conditions in which they are kept before inoculation. When bean plants of two ages were inoculated with the same inocula $(1.2 \mathrm{mg}$. $/ \mathrm{l}$. of TNVb mixed with different amounts of $\mathrm{SV}$ ), the large lesions expressed as a percentage of the small were $5,18,59$ in young plants and 9,38 and 138 in old plants, respectively, for $2 \cdot 4,0 \cdot 6$ and $0 \cdot 15 \mathrm{mg}$./l. of $\mathrm{SV}$, suggesting that, as plants age, their resistance to infection by $\mathrm{SV}$ increases more than to TNVb.

The susceptibility of the plants to virus infection can be increased by keeping them at $36^{\circ}$ for a day or two before inoculation (Kassanis, 1952). To see whether their preinoculation treatment affects the interaction between the two viruses, bean plants were kept for one day at $36^{\circ}$ or $18^{\circ}$ and then inoculated on opposite half leaves with TNVb alone and TNVb mixed with SV $(0.25 \mathrm{mg}$. $/$. for each virus $)$. The plants pretreated at $36^{\circ}$ produced about twice as many lesions as plants kept at $18^{\circ}$ before inoculation. The prescnce of SV increased the total number of lesions on both lots of plants, by $42 \%$ in plants pretreated at $36^{\circ}$ but by only $17 \%$ in plants kept at $18^{\circ}$, suggesting that the pretreatment increases susceptibility to infection by SV more than by TNVb. The interaction between SV and TNVb also depends on the species of plant. In Nicotiana glutinosa L. lesions look the same whether inoculated with $\mathrm{TNVb}$ alone or mixed with SV. When bean and $N$. glutinosa plants were inoculated on opposite half leaves with TNVb alone $(0.6$ and $6 \mathrm{mg} . / \mathrm{l}$., respectively, for the two species) or together with $\mathrm{SV}$ (10 mg./l.), the two inocula produced the 
same number of lesions in beans, whereas in $N$. glutinosa the mixed inoculum produced on average only $14 \%$ of the number of lesions produced by TNVb alone. Hence concentrations of $S V$ that do not affect the number of lesions produced by TNVb in beans have a large effect in $N$. glutinosa.

Table 2. Numbers and ratios of large and small lesions when mixed inocula $(T N V b$ and $S V$ ) are inoculated at different dilutions

Size and number of lesions on 6 half-leaves of French bean

Inocula $\overbrace{\text { Large }}^{\text {Sarge/small }}$

Purified preparations (TNVb $0.8 \mathrm{mg} . / \mathrm{l}$. and SV $0.6 \mathrm{mg} . / \mathrm{l}$.)

$\begin{array}{lrrr}\text { Endiluted } & 107 & 50 & 2 \cdot 1 \\ \text { Diluted } 1 / 2 & 82 & 16 & 5 \cdot 1 \\ \text { Diluted } 1 / 4 & \mathbf{3 5} & 1 & 35 \cdot 0 \\ \text { Diluted } 1 / 8 & 20 & 0 & -\end{array}$

Sap from tobacco plants inoculated with the Rothamsted culture

Diluted $1 / 5$

Diluted $1 / 25$

I iluted $1 ; 125$

Diluted 1/625

Diluted 1/312.5

$\begin{array}{lrl}(\mathrm{TNVb}+\mathrm{SV}) & & \\ 32 & 403 & 0 \cdot 08 \\ 19 & 85 & 0 \cdot 22 \\ 13 & 17 & 0 \cdot 76 \\ 3 & 1 & 3 \cdot 0 \\ 1 & 0 & -\end{array}$

Table 3. Numbers and ratios of large to small lesions with mixed inocula containing various concentrations of tobacco necrosis virus $(T N V b)$ and constant amounts of satellite virus $(S V)$ at $0.6 \mathrm{mg} . / \mathrm{l}$.

\begin{tabular}{cccc}
$\begin{array}{c}\text { Concentration of } \\
\text { large vims } \\
\text { (mg.jl.) }\end{array}$ & $\begin{array}{c}\text { Size and number of lesions on } \\
8 \text { half-leaves of French bean }\end{array}$ & \\
\cline { 2 - 3 } 8.8 & Iarge & Sinull & Largeismall \\
$4 \cdot 4$ & 53 & 427 & $0 \cdot 12$ \\
$2 \cdot 2$ & 4.7 & $27: 3$ & $0 \cdot 17$ \\
$1 \cdot 1$ & 2.2 & 170 & $0 \cdot 13$ \\
& 7 & 46 & 0.15
\end{tabular}

When a constant amount of 'TNVa or TNVb was mixed with decreasing amounts of $\mathrm{SV}$, the ratio of large to small lesions in beans increased (Fig. 1). The ratio of large to small lesions also increased when a mixture of TNVb and SV was inoculated at different dilutions (Table 2). Hence the effect on lesion size depends not only on the ratio of the two viruses in the inoculum but also on the absolute concentration of SV; dilution presumably lessens the chances of TNVb and SV particles mecting in the same cell of infection (multiple infection) and interfering with each other. When beans were inoculated with $\mathrm{TNV}$ b at different concentrations in a constant concentration of $\mathrm{SV}$, the ratio of large to small lesions remained about the same (Table 3). Fewer lesions were produced by the diluted TNVb; but at a constant concentration of SV the chances that $\mathrm{INVb}$ and $\mathrm{SV}$ particles will meet in the same cell are also unchanged.

In all the above experiments TNVb and SV were inoculated simultaneously as 
mixtures, but lesion size can be affected when the two are inoculated at different times. When TSVb was inoculated first, however, SV had to be inoculated soon afterwards to affect lesion size. About half the lesions were small when the interval between the two inoculations was 30 min., about one-quarter when the interval was 5 hr. and all lesions were large when the interval was 1 day. By contrast, bean plants inoculated with $40 \mathrm{mg}$. 1 . of $S \mathrm{~V}$ produced only small lesions when re-inoculated with $\mathrm{TNVb} 5$ days later.

\section{Interferene in virus multiplication}

TNVb multiplies less extensively in leaves inoculated with a mixture of the two viruses than when inoculated alone; the extent to which its multiplication is depressed depends on the ratio of the two viruses in the inoculum. With inocula containing much more $\mathrm{SV}$ than $\mathrm{TNVb}$, the amount of $\mathrm{TNVb}$ was too small to be detected serologically (Table 4). Other factors than the ratio of the two viruses in the inoculum can influence the virus concentration. SV reached higher concentrations in bean than in tobaeco leaves, whereas TNVb inoculated alone reached higher concentrations in tobaceo than in bean (Table 5). As SV seens completely dependent on 'TNVb it is interesting that it multiplies better in the species of plant less well suited for the multiplication of TNVb. In both bean and tobacco, TNVb was more effective than TNVa in stimulating the multiplication of SV.

Table 4. Concentration of tobacco necrosis virus $(T N V b)$ and satellite virus $(S V)$ in single and mixed infections in tobacco plants, 6 days after inoculation

\begin{tabular}{lcr} 
& \multicolumn{2}{c}{$\begin{array}{c}\text { Reciprocals of precipitation } \\
\text { end point }\end{array}$} \\
\cline { 2 - 3 } Inocula* (mg./l.) & 16 & $\mathrm{SV}$ \\
TNVb 9 & 8 & 0 \\
TNVb 9 + SV 3 & 2 & 32 \\
TNVb $9+$ SV 9 & 0 & 16 \\
TNVb 9+SV 27 & & 16 \\
* 'Celite' was added in the inocula.
\end{tabular}

'Table 5. Comparison of the concentration of the two tobarco necrosis viruses (TNVa and $T N(b)$ when inoculated alone or together with $S V$ in bean and tobacco plants

\begin{tabular}{|c|c|c|c|c|}
\hline \multirow[b]{3}{*}{ Inoculia } & \multicolumn{4}{|c|}{ Reciprocals of presipitation end-point } \\
\hline & \multicolumn{2}{|c|}{ Bean } & \multicolumn{2}{|c|}{ Tobucco } \\
\hline & TVNa or $b$ & sV & TNVa or $b$ & $\mathbf{S V}$ \\
\hline TNVb* & 4 & 0 & 16 & 0 \\
\hline $\mathbf{T N V b}+\mathbf{S V}$ & 0 & 128 & $\mathbf{1}$ & 16 \\
\hline TNVa & 2 & 0 & 8 & n \\
\hline $\mathrm{lNV} \mathbf{\mathrm { a }}+\mathrm{SV}$ & $\mathbf{0}$ & 32 & 0 & 4 \\
\hline
\end{tabular}

* T.NVb and TNVa were at $12 \mathrm{mg.jl}$ and SV at $81 \mathrm{mg} . / \mathrm{l}$. The tobacco plants were inoculated with the aid of 'Celite'.

Concentrations of $\mathrm{TNVb}$ and $\mathrm{SV}$ were affected differently by changing temperature. Although TNVb alone and SV (in plants with both viruses) reached maximum concentration in tobacco plants kept at about $20^{\circ}$, the concentration of SV de- 
creased relatively more than that of $\mathrm{TNVb}$ when the temperature was raised to $26^{\circ}$. The multiplication of $\mathrm{TNVb}$ was inhibited by $\mathrm{SV}$ more at lower than at higher temperatures, which agreed with the effects of temperature on the type of lesion. At $26^{\circ}$ all the lesions formed by mixed inocula were large ('lable 1 ).

The multiplication of the two viruses depended on whether they were inoculated simultancously or not. When SV was inoculated together with, or 1 day before, TNVb, SV multiplied more than when inoculated after TNVb. The multiplication of ' $\mathrm{TNVb}$ was decreased more by SV when the two viruses were inoculated together than when TNVb was inoculated 1 day before SV. When 'TNVb was inoculated one day before SV all the lesions were large, but SV nevertheless multiplied under these conditions (Table 6).

Table 6. Concentration of tobacco necrosis virus $(T N V b)$ and satellite virus ( $S V$ ) inoculated at different times to tobacco plants

\begin{tabular}{lccc} 
Sirst inoculation & Second inoculation & \multicolumn{2}{c}{$\begin{array}{c}\text { Reciprocal of precipitation } \\
\text { end-point }\end{array}$} \\
3 Jan. TNVb* & 4 Jan. SV & 16 & $\mathrm{SV}$ \\
3.Jan. TSVb & 4 Jan. Water & 32 & $\mathbf{8}$ \\
2 Jan. SV & 3 Jan. TNVb & 8 & $\mathbf{3 2}$ \\
2 Jan. Water & 3 Jan. LNVb & 8 & 0 \\
3 Jan. TNVb+SV & - & 2 & 32
\end{tabular}

* TNVb and SV were used at $10 \mathrm{mg} . / 1$. ; 'Celite' was added in all the inocula.

\section{Properties of $S V$}

Purification. Most of the TNVb present in purified preparations of the mixed Rothamsted culture could be precipitated by ammonium sulphate at 10 to $15 \%$ saturation leaving about $75 \%$ of $\mathrm{SV}$ in the supernatant fluid, as shown by scrological and infectivity tests. The average numbers of lesions per leaf were 61,89 and 5 , respectively, with the mixture, with the precipitate (containing most of the TXVb), and with the supernatant fluid (containing most of the SV). To remove the remaining $\mathrm{TNVb}$ the preparations of $\mathrm{SV}$ were centrifuged in sucrose gradients. Purified SV inhibited the lesions formed by $\mathrm{TNVb}$ at concentrations as low as $1 / \mu . / l$. Concentrations less than $3 \mathrm{mg} . / \mathrm{l}$. were not precipitated by homologous antiserum.

Purified preparations of SV scattered light much less than preparations of the same concentration of 'TNVa or TNVb, and when centrifuged at $75,000 \mathrm{~g}$ for $3 \mathrm{~h}$. they formed crystalline pellets. Pillcts freslly suspended in water were turbid and contained many crystals of different sizes, most of which dissolved in $24 \mathrm{hr}$. at $4^{\circ}$. Under the microscope the crystals apprared as rhombic plates, and electron micrograph mensurements showed them to consist of particles the size of $\mathrm{SV}$ (PI. 1, fier. 1). The rhombic plates resembled the small crystals photographed by Bawden \& Piric (1915, Jig. 3) from concentrated purified preparations of the Rothamsted culture left for some wecks at $0^{\circ}$.

A preparation of SV twice separated in sucrose gradient columns contained $2 \cdot 0 \%$ phosphorus, in agreement with the results of Biswden \& Piric (1942) who found the phosphorus content of all tobacco necrosis virus isolates to be between 1.7 
and $2.0 \%$. The particle of $\mathrm{SV}$ is one-third to one-half that of $\mathrm{TNVb}$ and therefore contains correspondingly less nucleic acid; this has been suggested as a possible reason for its apparent inability to multiply unaided (Kassanis \& Nixon, 1961). Since then, brome grass mosaic virus has been reported to contain the smallest infective nucleic acid, with a molecular weight of $1 \times 10^{6}$ (Bockstahler \& Kaesberg, 1961). The equivalent molecular weight of SV calculated from the sedimentation constant $\left(50 \mathrm{~S}\right.$ ) is $1.85 \times 10^{6}$ (Bawden \& Pirie, 19.5), and of its nucleic acid $0.37 \times 10^{6}$, which is much smaller than the brome grass mosaic virus.

Particle size. Measurements of the diameter of SV particles were from $14 \cdot 3$ to $21.2 \mathrm{~m} \mu$ according to the technique used for mounting the preparations (Kassanis \& Nixon, 1961). Because of the uncertainty about the true size of SV, rows of particles were measured on several electron micrographs of the carbon copies of shadowed erystals of $\mathrm{SV}$. The result depended on which direction in the crystal the neasurement was made; one way gave the diameter as 14.4 and the other $16.7 \mathrm{~m} \mu$. With a diameter of $16.7 \mathrm{~m} \mu$ and 1.35 density (the density of bushy stunt virus) the particles would have a weight equivalent to a molecular weight of $1.9 \times 10^{8}$, which is close to that calculated from sedimentation constant (Bawden \& Pirie, 1945). Hence $16 \cdot 7 \mathrm{~m} \mu$ is possibly the actual diameter of $S V$.

Table 7. Inactivation of satellite virus by heat

$20 \mathrm{mg} . / \mathrm{l}$. of virus in $0.5 \mathrm{ml}$. lots were heated for $10 \mathrm{~min}$. at different temperatures and mixed with equal volumes of tobacco necrosis virus (TNVb) at $1.2 \mathrm{mg} . \mathrm{ll}$.

$\begin{array}{cccc} & \begin{array}{c}\text { Size and number of lesions on } \\ 8 \text { French bean half-leaves }\end{array} & \text { Survival } \\ \text { Treatment } & \text { Large lesions } & \text { Small lesions } & (\%) \\ \text { Lnheated } & 0 & 170 & - \\ \text { Heated at } 70^{\circ} & 21 & 128 & 8 \\ 80^{\circ} & 47 & 114 & 4 \\ 85^{\circ} & 95 & 169 & 2 \\ 85^{\circ} & 195 & 25 & 1 \\ 95^{\circ} & 199 & 0 & 0\end{array}$

Inactivation by heat. SV was heated for $10 \mathrm{~min}$. at different temperatures. After heating it was mixed with constant amounts of TNVb, inoculated to beans, and the large and small lesions counted separately. A few small lesions were formed with SV heated at $90^{\circ}$ but none after heating at $95^{\circ}$ ('Table 7). Serological tests on the sap from inoculated leaves confirmed that SV heated at $95^{\circ}$ did not multiply. Heating for $10 \mathrm{~min}$. at $70^{\circ}$ destroyed over $90 \%$ of the infectivity, whereas heating at $90^{\circ}$ did not alfect the ability to precipitate with antiserum. Loss of infectivity without obvious change of the precipitation titre is a feature of many spherical viruses. TNVb lost inlectivity in $10 \mathrm{~min}$. between $75^{\circ}$ and $80^{\circ}$, whether heated alone or with SV.

Stability. The activity of purified preparations of SV stored at $3^{\circ}$ remained unaltered for several months. That some activity remained after 17 years was shown by tests on several purified preparations of the Rothamsted culture of TNV given to me by Mr N. W. Pirie. These had been left at $3^{\circ}$ since 1943 , and produced no lesions on bean but when mixed (at $50 \mathrm{mg}$./l.) with active $\mathrm{TNVb}$ they decreased the size of 
$\mathbf{2 5} \%$ of the lesions. A 17 -year-old preparation of the 'potato' isolate of TNV contaminated with the Rothamsted culture of TNV (Bawden \& Pirie, 1942) was also inoculated to beans; it produced a few lesions and virus from these was multiplied in tobacco. Partially purified virus prepared from the tobacco contained mostly large particles but also some small ones. The presence of SV suggests that the 'potato' isolate, a stable tobacco necrosis virus (Bawden \& Pirie, 1942), is another that can activate SV.

Ultraviolet inactivation. T.NVb is inactivated 2 times faster than $\mathrm{SV}$ by ultraviolet irradiation at a wavelength of $25+\mathrm{m} / \mathrm{\text {(Fig. }} 2$ ).

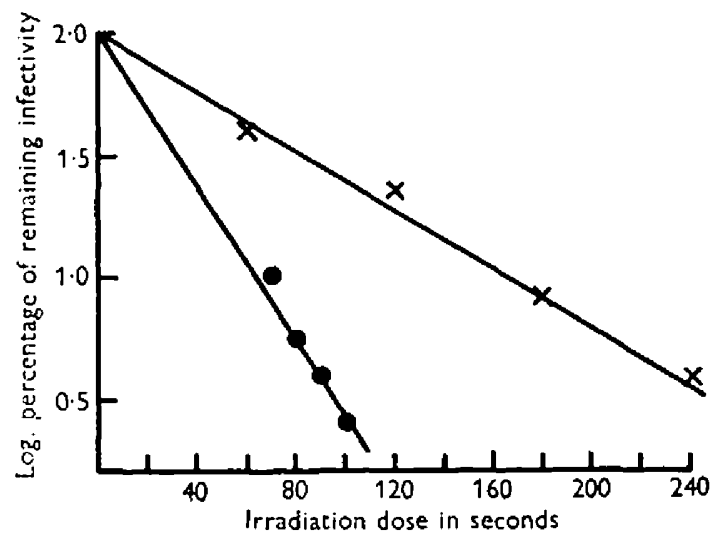

Fig. 2. The inactivation of SV and TNVb, (both $20 \mathrm{mg} . / \mathrm{I}$.) by ultraviolet light. $20 \mathrm{~cm}$. from source in films $0.07 \mathrm{~cm}$ deep. $x-x, \mathrm{SV} ;-0, \mathrm{TNV}$.

Ultraziolet absorption. The ultraviolet absorption spectrum of SV was similar to that of $\mathrm{TNVb}$, with a maximum at $260 \mathrm{~m} \mu$ and a minimum at $241 \mathrm{~m} \mu$. The extinction coelficient for $\mathrm{SV}$ was 6.5 (for $1 \mathrm{mg}$. $/ \mathrm{ml}$.).

Electrophoretic mobility. In $0 \cdot 066 \mathrm{~m}$-phosphate huffer (pH 7.0) SV was stationary even after 3 hr. at a potential gradient of $9.5 \mathrm{v} . / \mathrm{cm}$., whereas TNVb had a mobility of $-7 \cdot 4 \times 10^{-5}\left(\mathrm{~cm}^{2} \mathrm{sec}^{-1} \mathrm{v} .^{-1}\right.$. The difference in mobility between the two viruses was found useful in separating them from purified preparations of the Rothamsted culture. SV remained in the descending arm and after $2 \frac{1}{2} \mathrm{hr}$. at a potential gradient of $9.5 \mathrm{v} / \mathrm{cm}$., TNVb moved away and the arm was disconnected. Preparations of the Rothamsted culture usually have much less TNVh than $\mathrm{SV}$ and electrophoresis gave clcan preparations of $\mathrm{SV}$. The two viruses were also separated from a preparation of the Rothamsted culture enriched by adding TNVb so that the mixture contained per weight more of TNVb than SV. SV prepared from this run was almost free from TNVb particles ('Table 8 ). Preparations of $\mathrm{SV}$ obtained by electrophoresis from ordinary preparations of the Rothamsted culture were as free from TNVb as those obtained by centrifugation in surrose gradient columns. Separation by electrophoresis was preferred because it was less wasteful, and its efficiency was not impaired by high virus concentration or by aggregated SV in the mixture. Purified preparations of the Rothamsted culture containing aggregated SV gave three zones when centrifuged in sucrose gradients; the lowest zone was difficult to remove from the tube through the middle zone containing $\mathrm{TNVb}$ (Kassanis \& Nixon, 1961). 
Nucleic acid of $S V$. When preparations of SV were disrupted with phenol (Geircr \& Schramm, 1956) they were still infective and still gave many of the phenomena shown by intact virus. Such disrupted preparations were inactivated in less than a day at $20^{\circ}$. 'They presumably consisted largely of nucleic acid, for they were rapidly inactivated by dilute pancreatic ribonuclease, which does not inactivate intact virus. Fresh preparations of nucleic acid did not produce lesions when inoculated alone to bean or tobacco and no virus was later recoverable from such inoculated plants. When inoculated together with TNVb, however, these nucleic

Table 8. Separation of satcllite virus (SV) from a mixture with $\mathrm{TNVb}$ by electrophoresis

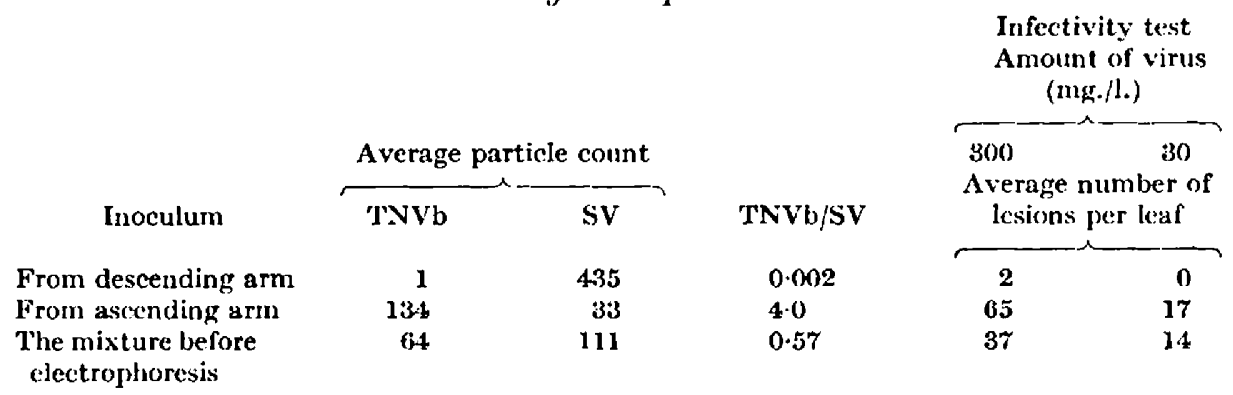

acid preparations decreased the size of the lesions and such inoculated plants later contained normal SV. To influence the size of lesions produced by $\mathrm{TNVb}$, the nucleic acid and TNVb had to be inoculated simultaneously or the TNVb within an hour or so of the nucleic acid. This is a striking difference from the intact virus which produces its effects when inoculated several days before the TNVb. The difference between the behaviour of the nucleic acid and intact virus presumably largely reflects the grcater instability of the nucleic acid. There was, however, some result from an early inoculation with nucleic acid, for although the lesions were all large some SV could later be extracted from such plants. In two experiments out of five, when $\mathrm{TNVb}$ was inoculated as long as 2 days after nucleic acid, sap extracted 6 days later contained sume SV, although only about $6 \%$ of the amount from leaves inoculated with TNVh immediately after nucleic acid. In the other three experiments of this kind, SV was not detected when the inoculation with TNVb was delaycd for 2 days. Different glasshouse temperatures may have caused the inconsistency. Since the nucleic acid preparations lost their infectivity within a day at $20^{\circ}$, it seemed that in vivo some nucleic acid was either protected from inactivation by cellular components, or had proxluced an undetectable amount of new virus at some sites, where multiplication reached detectable amounts after infection with ' $\mathrm{T} N \mathrm{~N}$. $\mathrm{TNVb}$ when inoculated with the nucleic acid of SV, as with whole SV, sometimes produed twice as many lesions as when inoculated on its own.

\section{DISCUSSION}

The interactions between TNVb and SV seem to difler from the various types already described. There is nothing novel in the multiplication of $\mathrm{TNVb}$ being decreased by SV, except that such interference is usually between related strains, whereas TNVb and SV arc serologically unrelated. However, tobacco severe etch 
virus can suppress the multiplication of potato virus $Y$, which is not related to it (Bawden \& Kassanis, 1945). Nor is there anything new in the idea that one virus mity increase the amount to which another multiplies; but the previous examples have all been with viruses clearly able to multiply on their own (Rochow \& Ross, 1955; Kassanis, 1961). The striking fenture of the interaction between TNVb and SV is that SV apparently depends completely on TNVb for its ability to multiply. However, it is necessary to say apparently, for although when inoculated alone to plants it is not pathogenic and it does not multiply detectably, it is neccssary to consider the limitations of the techniques used in detection. The most sensitive technigue is the ability to affect the size of lesions produced by TNVb and this can be done by preparations containing as little as $1 / \mathrm{gg} . / \mathrm{l}$. Serological tests are very much less sensitive than infectivity tests : the minimum amount detectable is $3 \mathrm{mg} . / 1$. Obviously, the $\mathrm{SV}$ could multiply many times before it became detectable serologically and cven by infectivity tests. Attempts were made to demonstrate multiplication in leaves inoculated with SV alone by extracting the sap 10 day's later and mixing it with TNVb and inoculating to beans, but all the lesions were large.

There are three reasons for suspecting that $S V$ induces changes in the plant cells and so withstands inactivation, or perhaps multiplies to a limited extent. First, plants inoculated many days earlier with $\mathrm{SV}$ still contain considerable amounts of SV when later re-inoculated with TNVb. This could be explained simply by particles from the initial inoculum surviving intact for days in the inoculated leaves until their replication is sct going by T.NVb. However, the idea conflicts with the behaviour of other viruses as stable as $\mathrm{SV}$, which arc inactivated within a few days of being inoculated in hosts where they do not multiply. Secondly, under certain conditions, the infectivity of TNVh is greater when inoculated with SV or its nucleic acid, than when inoculated on its own: some particles of TNVb which otherwise could not have infected can do so in the presence of SV. This suggests that SV changes eells so they become more susceptible to TNVb. Thirdly, and perhaps more important, is the fact that some SV can be recovered from plants inoculated with SV nucleic acid 2 days before they are inceulated with TNVb, for it seems highly improbable that the fragile nucleic acid could survive unharmed for 2 days while waiting to be activated by $\mathrm{TNV}$. It may be protected from inactivation by association with some host component, perhaps by entering a state comparable to that of bacteriophage in lysogenic bacteria, or it may have initiated virus multiplication which has proceeded only to an undetectable degree.

These are possibilitics, but the main picture from the results is one of SV being completely dependent on an attendant virus to create conditions in which it can multiply. The attendant virus secms to lose from the association, because it multiplics less in doubly-infected leaves than on its own. The onc suggestion of gain is that occasionally more infection occurs with inocula of $\mathrm{TNVb}+\mathrm{SV}$ than with inocula of TNVb alone. However, cren when this happens most of the lesions are smaller than they would be with 'TNVb alone and at each infection site its multiplication is restricted.

I am indebted to Dr A. Kleckzkowski for the electrophoresis and Mr R. D. Woods for the electron microscopy. 


\section{REFERENCES}

Bawden, F. C. \& Kassanis, B. (1945). The suppression of one plant virus by another. Ann. appl. Biol. 32, 52.

Bawden, F. C. \& Pinie, N. W. (1942). A preliminary description of preparations of some of the viruses causing tobacco necrosis. Brit. J. exp. Path. 23, 314.

Bawden, F. C. \& Pirie, N. W. (1945). Further studies on the purification and properties of a virus causing tobacco necrosis. Brit. J. exp. Path. 26, $27 \%$.

Bawden, F. C. \& Pinir, N. W. (1950). Some factors affecting the activation of virus preparations made from tobacco leaves infected with a tobacco necrosis virus. $J$. gen. Microbiol. 4, 464.

Bockstahler, L. E. \& Kaesberg, P. (1961). Bromegrass mosaic virus : a virus containing an unusually small ribonucleic acid. Nature, Lond. 190, 192.

Griner, A. \& Schramm, G. (1950). Infectivity of ribonucleic acid from tobacco mosaic virus. Nature, Lond. 177, 702.

Kassanis, B. (1952). Some effects of high temperature on the susceptibility of plants to infection with viruses. Ann. appl. Biol. 39, 358.

Kassanis, B. (1961). The transmission of potato aucuba mosaic virus by aphids from plants also infected by potato viruses $A$ or $Y$. Virology, 13,93.

Kassanis, B. \& Nixon, H. L. (1960). Activation of one plant virus by another. Nature, Lond. 187, 713.

KasSanis, B. \& Nrxon, H. I. (1961). Activation of one tobacco necrosis virus by another. J. gen. Microbiol. 25, 459 .

Rocrow, W. F. \& Ross, A. F. (1955). Virus multiplication in plants doubly infected with potato viruses $\mathrm{X}$ and $\mathrm{Y}$. Virology, 1, 10.

\section{EXPLANATION OF PLATE}

Crystal of staellite virus (platinum shadowed carbon replica). $\times 54,000$. 


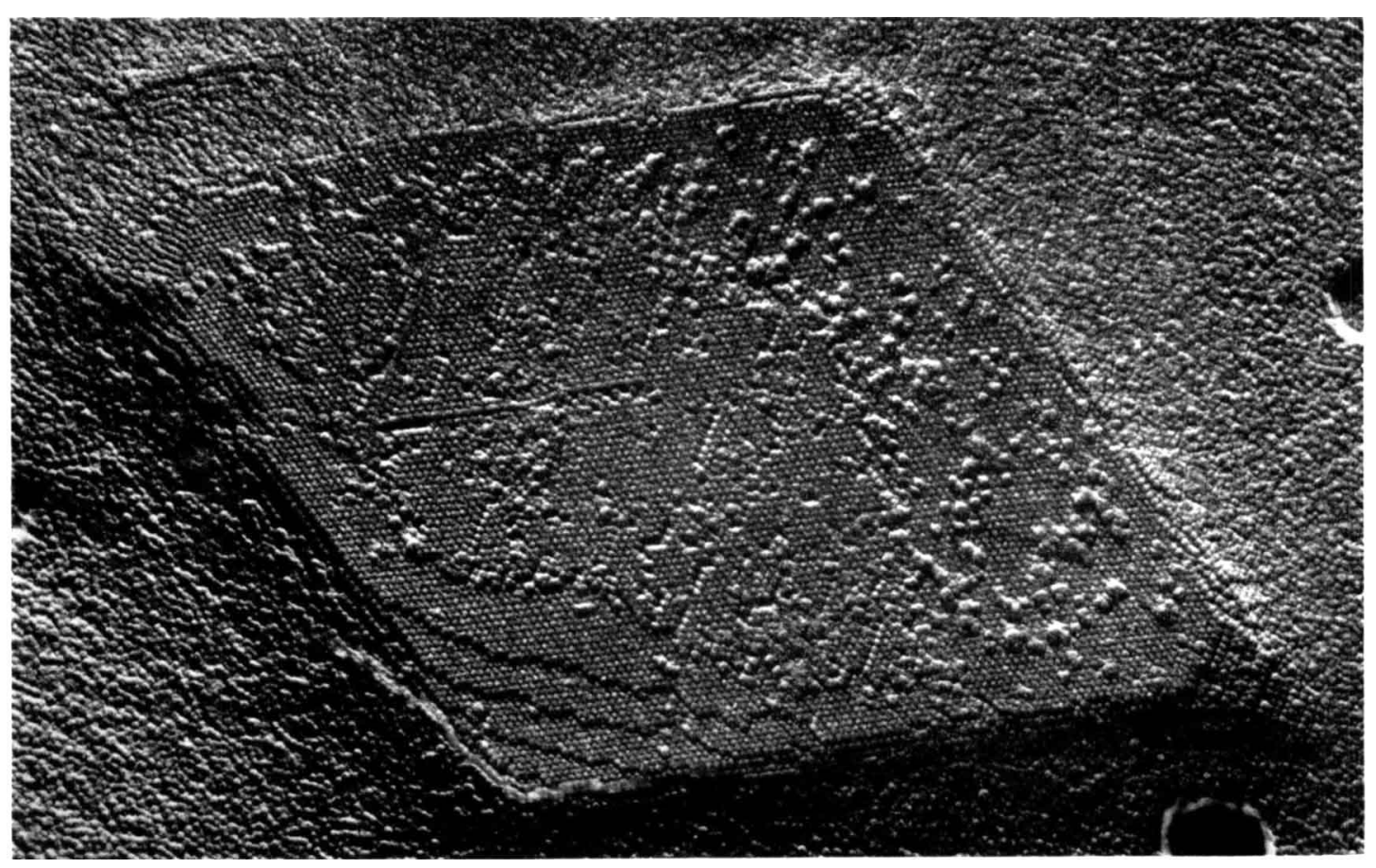

